\title{
Of flies, mice and men: a systematic approach to understanding the early life origins of chronic lung disease
}

\author{
Susanne Krauss-Etschmann, ${ }^{1,2}$ Andrew Bush, ${ }^{3}$ Saverio Bellusci, ${ }^{4}$ Guy G Brusselle, ${ }^{5}$ \\ Sven Erik K Dahlén, ${ }^{6}$ Stefan Dehmel, ${ }^{1}$ Oliver Eickelberg, ${ }^{1}$ Greg Gibson, ${ }^{7}$ Machteld \\ N Hylkema, ${ }^{8}$ Petra Knaus, ${ }^{9}$ Melanie Königshoff, ${ }^{1}$ Clare M Lloyd, ${ }^{10}$ Paolo Macciarini, ${ }^{11}$ \\ Arnaud Mailleux, ${ }^{12}$ Benjamin J Marsland, ${ }^{13}$ Dirkje S Postma, ${ }^{14}$ Graham Roberts, ${ }^{15,}{ }^{16}$ \\ Christos Samakovlis, ${ }^{17}$ Janet Stocks, ${ }^{18}$ Joke Vandesompele, ${ }^{19}$ Matthias Wjst, ${ }^{1}$ \\ John Holloway ${ }^{16}$
}

For numbered affiliations see end of article

\section{Correspondence to} Dr Susanne Krauss-Etschmann, Comprehensive Pneumology Center, Helmholtz Center Munich and Children's Hospital of Ludwig-Maximilians University, Max-Lebsche Platz 31, Munich 81377, Germany; susanne.krauss-etschmann@ helmholtz-muenchen.de

Received 13 March 2012 Accepted 30 April 2012 Published Online First 10 July 2012
To cite: KraussEtschmann S, Bush A, Bellusci $S$, et al. Thorax 2013;68:380-384.

\section{ABSTRACT}

Despite intensive research efforts, the aetiology of the majority of chronic lung diseases (CLD) in both, children and adults, remains elusive. Current therapeutic options are limited, providing only symptomatic relief, rather than treating the underlying condition, or preventing its development in the first place. Thus, there is a strong and unmet clinical need for the development of both, novel effective therapies and preventative strategies for CLD. Many studies suggest that modifications of prenatal and/or early postnatal lung development will have important implications for future lung function and risk of CLD throughout life. This view represents a fundamental change of current pathophysiological concepts and treatment paradigms, and holds the potential to develop novel preventative and/or therapeutic strategies. However, for the successful development of such approaches, key questions, such as a clear understanding of underlying mechanisms of impaired lung development, the identification and validation of relevant preclinical models to facilitate translational research, and the development of concepts for correction of aberrant development, all need to be solved. Accordingly, a European Science Foundation Exploratory Workshop was held where clinical, translational and basic research scientists from different disciplines met to discuss potential mechanisms of developmental origins of CLD, and to identify major knowledge gaps in order to delineate a roadmap for future integrative research.

\section{DEVELOPMENTAL ORIGINS OF RESPIRATORY HEALTH AND DISEASE: THE CONCEPT}

Broad interest in developmental processes, and their relationship to later human health, emerged in 1986 when Barker et al demonstrated a positive geographical correlation of infant mortality with adult death rates from coronary heart disease in England and Wales. ${ }^{1}$ The 'Barker hypothesis' proposed that low birth weight, as a proxy for intrauterine nutrient restriction, might be the major determinant for cardiometabolic disease in adulthood. ${ }^{2}$ This concept was further fuelled by retrospective observations from the 'Dutch Hunger Winter' in 1944, where severe undernourishment during pregnancy was associated with increased susceptibility for obesity, arterial hypertension or type II diabetes in the offspring. ${ }^{3}$ Over time, it became clear that not only a highly deficient environment, but also other intrauterine exposures, such as environmental toxicants and maternal disease states are associated with later disease risks. Accordingly, the concept of 'Developmental Origins of Health and Disease' suggests that the fetus makes physiological adaptations in response to the intrauterine environment to prepare itself for postnatal conditions. These adaptations may be beneficial if intra- and extra-uterine environment correspond to each other, but will be disadvantageous if the postnatal environment has changed in the meantime, or has been wrongly 'predicted'. While these hypotheses have been mainly elaborated in relation to cardiometabolic diseases and behaviour, epidemiological studies suggest that the risk of developing chronic lung disease (CLD) is equally modified through perinatal exposures. This has been most convincingly shown for asthma ${ }^{4-6}$ and $\mathrm{COPD},{ }^{7-9}$ where a number of factors that are either protective, or predispose to the disease, have been identified. It is suspected that these modifications are, at least in part, mediated by epigenetic mechanisms. ${ }^{10}$ This is important, as epigenetic modifications have been shown to be transmitted trans-generationally, ${ }^{11}$ suggesting that 'memory' of previous environmental exposure in a population may result in increased disease risk in subsequent generations. Moreover, the importance of epigenetic processes also provides an opportunity for intervention, as animal models have shown, that interventions such as maternal dietary modification in pregnancy have the potential to modify epigenetic programming and reduce subsequent disease risk. ${ }^{12}$ Given all of this, we would like to propose a Developmental Origins of Respiratory Health and Disease (DORHaD) concept to focus activity on an area with the potential to deliver improved respiratory health outcomes.

However, the extent to which disease risks are established during embryo-fetal life and the underlying mechanisms are far from understood, and susceptible developmental windows have not been adequately explored. If interventions that will improve the health of the population with respect 
to CLD are to be developed, it is essential that these knowledge gaps are addressed. To this end, a multidisciplinary team involving experts from developmental biology, immunology, cell signalling, lung biology, stem cell biology, (epi)genetics, epidemiology and clinical science, met in an ESF-Exploratory Workshop (http://www.esf.org/activities/exploratory-workshops/ medical-sciences-emrc.html? year $=2011$ \&domain=EMRC) to develop a conceptual framework for future research on developmental origins of CLD. This review aims to demonstrate why and how interactions among clinical and basic research disciplines can generate a fruitful cross-fertilisation of ideas to treat and/or prevent CLD.

\section{LUNG DEVELOPMENT-AND WHAT HAPPENS IF IT GOES WRONG}

\section{Development and molecular pathways}

Mammalian lung development begins by formation of a bud from the floor of the primitive foregut around the fourth week of human gestation and continues through the pseudoglandular, canalicular, saccular and alveolar stages far into postnatal life. ${ }^{13}$ During this highly controlled process, the endoderm gives rise to the different epithelial cell lineages, while structural components of the lung, such as pulmonary vessels or airway smooth muscle, originate from the mesoderm. Once fully mature, the lung will harmonise the functions of around 40 different cell types. ${ }^{14}$ The formation of such a complex organ requires the precise spatiotemporal orchestration of multiple signalling molecules during development. Animal models of airway development demonstrate that early disruption of critical signalling pathways can lead to abnormal pulmonary phenotypes after birth. The first example for a phylogenetically conserved genetic program orchestrating airway branching derives from the Drosophila branchless $(\mathrm{Bnl})^{15}$ and its mammalian homologue, Fibroblast Growth Factor 10 (FGF10). Both, Bnl and FGF10, are expressed and secreted by the surrounding mesenchyme of the epithelial buds and control their outgrowth during early lung development through their epithelial receptors, Breathless and FGFR2-IIb, respectively. The crucial contribution of FGF10 signalling to branching morphogenesis is demonstrated by

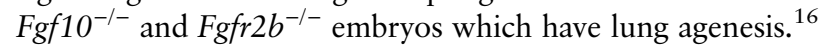

An entirely different level of regulation of lung development involves microRNAs (miRNAs) that suppress gene expression at the posttranscriptional level through binding of target mRNAs, and regulate essential cellular functions, such as growth, differentiation and programmed cell death. Hence, miRNAs have already been shown to control the development of broncho-alveolar tissues, ${ }^{17}$ and also respond to environmental factors, such as LPS ${ }^{18}$ or tobacco smoke, ${ }^{19}$ while others participate in epigenetic regulation. ${ }^{20}$ Nonetheless, it is largely unknown how microRNAs precisely regulate lung development or contribute to airway tissue remodelling.

\section{From developmental pathways to disease}

Through experimental disruption of developmental pathways, we now know that molecular pathways involved in lung development can be recapitulated later in life, provoking pathologic changes in complex respiratory diseases. For example, canonical Wnt/ $\beta$-catenin signalling has recently been identified as a key pathway for early lung development. ${ }^{21} \mathrm{Wnt}$ ligands and their receptors are expressed in a highly cell-specific manner in the developing lung and recede during later stages. In experimental and human fibrotic lung disease, the $\mathrm{Wnt} / \beta$-catenin signalling pathway is aberrantly reactivated, and has been linked in vitro and in vivo with alveolar epithelial cell repair mechanisms via one of its target genes Wnt1-inducible signalling pathway protein 1 (WISP1). ${ }^{22}$ In vivo depletion of WISP1 results in significant attenuation of lung fibrosis and improved survival in preclinical studies. Wnt-signalling genes have been further associated with impaired lung function in two childhood asthma cohorts, ${ }^{23}$ and activation of this pathway led to enhanced proliferation of bronchial epithelial cells. ${ }^{24}$ However, the precise contribution of each of the 19 Wnt ligands, 10 Frizzled receptors and signalling components to development and disease remains to be elucidated.

Studies in animal models and patients with pulmonary fibrosis or COPD have also provided evidence for a reactivation of other developmental pathways, such as TGF- $\beta$-, or PTEN/ PI3kinase/Akt-mediated signalling. ${ }^{25}$

\section{From growth to lung function}

For normal lifelong lung function, the prerequisites are: (1) normal lung function at birth; (2) normal growth in lung function until the adult plateau at 20-25 years of age; and (3) no accelerated deterioration from the plateau. Cohort studies have established that lung function either tracks or deteriorates, but never improves, after the preschool years. ${ }^{26-28}$ Thus, lung function in adult life is critically dependent on in utero and postnatal lung development.

An important antenatal factor affecting newborn lung function is maternal smoking, which causes structural effects on the developing lung. ${ }^{29}$ A study in more than 13000 people showed that childhood disadvantage defined by either maternal or paternal asthma, childhood asthma, maternal smoking and childhood respiratory infections predicts worse adult lung function, a faster rate of lung function decline, and a greater prevalence of COPD. ${ }^{30}$ Other important influences include maternal antibiotic $^{31}$ and paracetamol ${ }^{32}$ use, maternal psychological stress, ${ }^{33}$ nutrition, ${ }^{34}$ diabetes, hypertension ${ }^{35}$ and exposure to pollution. $^{36}$

Equally important is to comprehend whether patterns of early somatic growth are associated with altered respiratory and immune development. Anthropometric measurements at birth, and markers of fetal growth, have been linked epidemiologically to asthma. ${ }^{37}$ In a recent large cohort study, longitudinal prenatal and infant growth patterns were related to wheeze and atopy at age 3 years in 1548 children. ${ }^{39}$ A rapid growth trajectory during 11-19 weeks of gestation followed by late gestation growth faltering was associated with atopy, suggesting that influences affecting fetal growth may also alter immune development. In contrast, a lower early fetal growth trajectory was associated with non-atopic wheeze, possibly reflecting an association with smaller airways.

With continuously improving survival rates, preterm birth is a further and increasingly important cause of early onset airflow obstruction. Recent evidence suggests that even non-ventilated late preterm infants from 33 weeks of gestation onwards show impaired lung function at least until the age of 8-9 years. ${ }^{40}$

In children, it is essential to distinguish between the effects of disease from those of growth and development. However, structural assessments of lung and airway dimensions do not necessarily reflect functional changes in lung growth and development, or vice versa. There are many tests of lung function, each interrogating a different airway region. For example, spirometry gives information about proximal airway disease, whereas, tests of gas mixing, such as lung clearance index (LCI) are more sensitive to distal airway problems. It is also not 
possible to distinguish alveolar number from alveolar size, as the cause of lung volume changes and measured lung volume changes may be artefactual due to airway disease. Thus, DLCO may give a measure of alveolar surface area, but the measurement is also vulnerable to impaired gas mixing. Determining whether there is disease in the silent regions of the lung (distal airways) or at the silent ages (2-6 years) is also a challenge. It is now possible to record flow volume curves in infancy (raised volume rapid thoracic compression technique), in pre-schoolers (using incentive spirometry) and from school age right through into old age (conventional spirometry). LCI can be performed at all ages, and after the first year of life, has the same normal range independent of age or height. This technique has been shown to be most sensitive in cross-sectional and longitudinal studies in cystic fibrosis, ${ }^{41}$ and preschool LCI is strongly predictive of school-age lung function abnormalities. ${ }^{42}$

In summary, tools for measuring lung function from birth to old age are available. However, while there is a good understanding of spirometry, it is relatively insensitive, in particular, to early airway disease. On the other hand, LCI is more sensitive, but we lack experience in interpreting changes. Nonetheless, suitable tools to monitor lung growth from birth onward are available and will help to identify clinical subphenotypes to ensure investigation of relevant outcomes, both, in humans and in preclinical studies.

\section{TOOLS OF THE TRADE-WHERE ARE WE NOW?}

As highlighted above, an increased understanding of the early life influences on lung development is needed to devise new strategies aimed at the primary prevention of lung disease. This requires development of cellular and animal models to investigate biological mechanisms and test effectiveness of preventative strategies.

Transgenerational studies in rodents have mainly focused on global maternal under/overnutrition, restriction of selective nutrients, psychological stress, or endocrine disorders, looking at cardiovascular and behavioural outcomes in some instances until the F2 generation. ${ }^{43}$ Evidence for transgenerational transmission of risk for lung disease emerged when offspring from tobacco smoke-exposed dams showed airway remodelling and decreased pulmonary expression of Wnt-signalling molecules and targets. ${ }^{44} \mathrm{~A}$ recent key study has demonstrated that nutritive supplementation of dams with methyl donors affects asthma risk across two subsequent generations, and involves differential methylation of several pulmonary genes including runt-related transcription factor 3 (Runx3) which, if not silenced, controls airway inflammation. ${ }^{10}$ Similarly, exposure of dams to diesel exhaust particles during gestation heritably alters innate immune responsiveness in F1 and F2 generations. ${ }^{45}$

As well as for prenatal exposures, appropriate postnatal challenge models are required. For example, the role of commensal bacteria in guiding the normal development of immune homeostasis has received attention, ${ }^{46}$ and neonatal allergen exposure models highlight how airway remodelling, inflammation and airway hyper-reactivity developed in parallel, rather than sequentially, ${ }^{47}$ supporting previous observations in humans. ${ }^{48}$ These observations underscore the importance of developing mouse models that are not only relevant to clinical disease phenotypes, but also address different developmental phases.

Despite the comparatively quick generation time of murine models and ample availability of molecular tools, refining of susceptible developmental windows for single types of exposures, and assessing risk transmission until F3/F4 generations is time consuming and expensive in mice.

The formation of the respiratory organ of the fruit fly, Drosophila melanogaster, demonstrates the basic principles of branch patterning and tube growth, and allows the analysis of airway maturation at the genome-wide level with or without perturbations and simplified interventions. Additionally, the transparency of fly embryos allows the analysis of cellular activities of airway epithelial cells in unprecedented detail by live imaging.

A comparative genomics approach among $D$ melanogaster and higher organisms can generate novel testable molecular pathways. These can then be studied in detail in higher organisms and during defined developmental stages. Such a preselection strategy would also justify efforts to move to large animal models where the anatomy and physiology of the lung, the placenta, pregnancy characteristics and dietary requirements have closer resemblance to humans. Additionally, 'high throughput' identification of relevant types of exposures might be feasible in lower organisms, such as D melanogaster.

Animal models need corresponding cell culture systems, which, although lacking the context of physiology, allow the biology of single molecules and pathways to be studied at high resolution. Available molecular tools are applicable also for two, triple or 3D culture systems to study the effects of intercellular crosstalk on cell-specific changes in gene or protein expressions in the context of exposures. These may be further complemented by whole-organ cultures, such as isolated ventilated perfused lungs or bronchial ring studies, to study a specific function in intact organs independent of the remainder organism, while maintaining the local physiological homeostasis. However, performing such analyses across species, and in combination with in vitro and in vivo disease models requires a considerable collaborative effort among researchers.

\section{WHY WE SHOULD TALK-A ROADMAP FOR FUTURE RESEARCH}

In understanding the mechanisms underlying $\mathrm{DORHaD}$, a major challenge will be the development of effective bridging systems to establish collaboration among basic and clinical sciences in order to combine expertise in human conditions with detailed knowledge of complementary model systems. In addition, computational biology is required to handle and analyse complex datasets in a systems biology approach.

The discovery and functional description of pathways during lung morphogenesis by developmental biologists have already led to the generation of hypotheses that are testable in translational studies, for example, knowledge of the role of developmental Wnt pathways in the pathobiology of pulmonary fibrosis has led to the identification of WISP1 as a potential therapeutic target.

Vice versa, reverse translation of clinical and epidemiological findings is mandatory to drive novel basic research. Thus, the identification of a number of maternal environmental exposures during pregnancy, as risk factors for adverse lung development, have formed the basis of mechanistic studies. ${ }^{49}$

These examples demonstrate that, while human clinical, genetic and environmental epidemiological studies can identify potential causes of disease, these will require the use of animal models to provide supporting evidence and to investigate biological mechanisms. Proving causality from early environmental exposures on development and disease will require a systematic, focused and concerted effort. A roadmap for future 


\section{A roadmap for joint research on early origins of chronic lung disease}

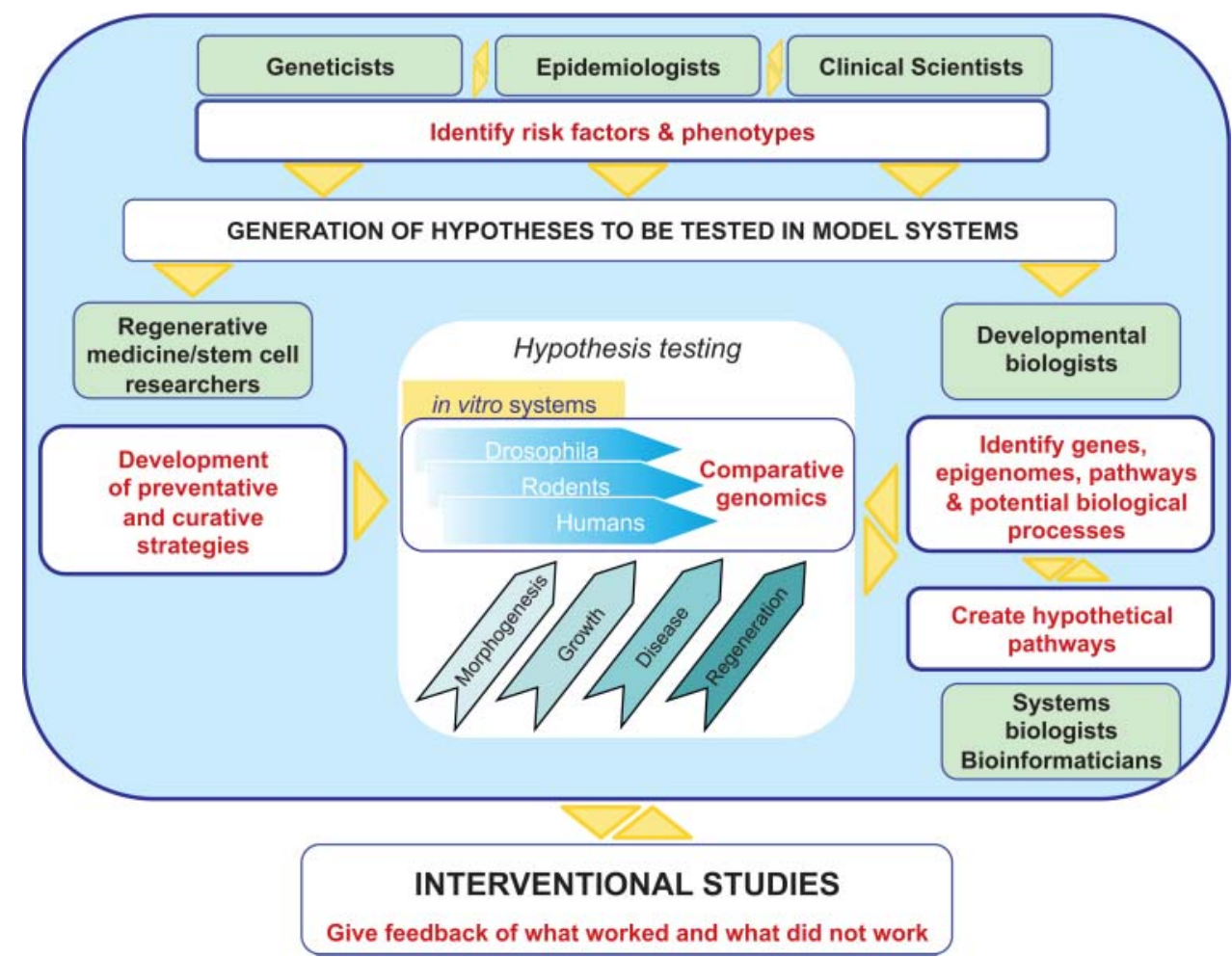

Figure 1 From observational studies, clinicians and epidemiologists create hypotheses to be tested in model systems. Developmental biologists discover molecular pathways and susceptible developmental windows relevant for later disease. This can lead to the identification of candidate treatment targets and testable hypotheses. A comparative genomics approach across model systems will allow to create hypothetical pathways and biological processes. Their role in development can again be tested in model systems. Researchers in regenerative medicine, such as stem cell biologists, work on practical applications that can be applied in interventional studies in the future.

\section{Box 1 Questions that can only be answered in a} concerted effort

- What is the normal ontology of relevant cell populations, and is their activity perturbed prior to the onset of disease?

- How do critical cell types and molecular events that initiate CLD respond to exposures?

- Which exposures affect lung development, how do they do so, and what are the crucial developmental time points?

- What are the underlying mechanisms of transgenerational transmission of disease risks, including molecular pathways, cellular interactions and epigenetic changes?

- What are relevant animal models that permit to track the risk for specific chronic pulmonary diseases across generations?

- What are triggers of normal development and of successful repair processes to regenerate lung tissue in the chronically injured lung?

- What is the effect of gender on developmental processes and subsequent risk of disease, and the underlying mechanisms?

- Which early life events affect the initial rate of increase and later rate of decline in lung function?

cross-disciplinary research is depicted in figure 1 . This, or similar approaches, should lead to answers to the most pressing questions (box 1) and will, hopefully, allow us to move on from experimental and observational studies to interventional cohort studies to prevent or cure CLD and deliver the promise of the DORHaD approach.

\section{Author affiliations}

${ }^{1}$ Comprehensive Pneumology Center, Helmholtz Center Munich, Munich, Germany

${ }^{2}$ Children's Hospital of the Ludwig Maximilians University, Munich, Germany

${ }^{3}$ Imperial College and Royal Brompton Hospital, London, UK

${ }^{4}$ Excellence Cluster in Cardio-Pulmonary Systems, Department of Internal Medicine II, University of Giessen Lung Center, Giessen, Germany

${ }^{5}$ Department of Respiratory Medicine, Laboratory for Translational Research in Obstructive Pulmonary Diseases, Gent University Hospital, Belgium

${ }^{6}$ Institute of Environmental Medicine, Division of Physiology, The Unit of

Experimental Asthma and Allergy Research, Karolinska Institutet, Sweden

${ }^{7}$ Center for Integrative Genomics, Georgia Institute of Technology, School of Biology,

Atlanta, Georgia, USA

${ }^{8}$ Department of Pathology and Medical Biology, University of Groningen, University

Medical Center Groningen, Groningen, The Netherlands

${ }^{9}$ Institute of Chemistry and Biochemistry, Freie Universitaet, Berlin, Germany

${ }^{10}$ Leukocyte Biology, National Heart \& Lung Institute, Imperial College London,

London, UK

${ }^{11}$ Department of General Thoracic and Regenerative Surgery and Intrathoracic

Biotransplantation University Hospital Careggi, Florence, Italy

${ }^{12}$ INSERM, U700, Université Paris Diderot, Sorbonne Paris Cité, Paris, France

${ }^{13}$ Faculty of Biology and Medicine, University of Lausanne, Service de Pneumologie, Lausanne, Switzerland

${ }^{14}$ Department of Pulmonology, University of Groningen, University Medical Center Groningen, Groningen, The Netherlands

${ }^{15} \mathrm{NIHR}$ Respiratory Biomedical Research Unit, University Hospital Southampton NHS Foundation Trust, Southampton, UK

${ }^{16}$ Human Development \& Health and Clinical \& Experimental Sciences, Faculty of Medicine, University of Southampton, Southampton, UK

${ }^{17}$ Developmental Biology, The Wenner-Gren Institute, Stockholm University, Sweden

${ }^{18}$ Portex Unit: Respiratory Physiology and Medicine, UCL Institute of Child Health, London, UK 
${ }^{19}$ Center for Medical Genetics, Gent University Hospital, Gent, Belgium and Biogazelle, Zwijnaarde, Belgium

Funding This publication was made possible by support from the European Science Foundation.GR is supported by the Wellcome Trust; GB is supported by Fund for Scientific Research, Flanders (Belgium); MH is supported by GABRIEL (a multidisciplinary study to identify the genetic and environmental causes of asthma in the European Community, contract No: 018996), and The Netherlands Asthma Foundation (NAF 3.2.09.036).

\section{Competing interests None.}

Provenance and peer review Not commissioned; internally peer reviewed.

\section{REFERENCES}

1 Barker DJ, Osmond C. Infant mortality, childhood nutrition, and ischaemic heart disease in England and Wales. Lancet 1986;1:1077-81.

2 Barker DJ, Winter PD, Osmond C, et al. Weight in infancy and death from ischaemic heart disease. Lancet 1989:2:577-80.

3 Heijmans BT, Tobi EW, Stein AD, et al. Persistent epigenetic differences associated with prenatal exposure to famine in humans. Proc Natl Acad Sci U S A 2008; 105:17046-9.

4 Devereux G, Turner SW, Craig LC, et al. Low maternal vitamin E intake during pregnancy is associated with asthma in 5-year-old children. Am J Respir Crit Care Med 2006;174:499-507.

5 Lannero $\mathrm{E}$, Wickman M, Pershagen $\mathrm{G}$, et al. Maternal smoking during pregnancy increases the risk of recurrent wheezing during the first years of life (BAMSE). Respir Res 2006:7:3.

6 Bekkers MB, Elstgeest LE, Scholtens S, et al. Maternal use of folic acid supplements during pregnancy and childhood respiratory health and atopy: the PIAMA birth cohort study. Eur Respir J 2012;39:1468-74.

7 Bush A. COPD: a pediatric disease. COPD 2008;5:53-67.

8 Hylkema MN, Blacquiere MJ. Intrauterine effects of maternal smoking on sensitization, asthma, and chronic obstructive pulmonary disease. Proc Am Thorac Soc 2009;6:660-2.

9 Svanes C, Omenaas E, Jarvis D, et al. Parental smoking in childhood and adult obstructive lung disease: results from the European Community Respiratory Health Survey. Thorax 2004;59:295-302.

10 Hollingsworth JW, Maruoka S, Boon K, et al. In utero supplementation with methyl donors enhances allergic airway disease in mice. J Clin Invest 2008;118:3462-9.

11 Skipper M. Epigenomics: epigenetic variation across the generations. Nat Rev Genet 2011:12:740.

12 Waterland RA, Jirtle RL. Transposable elements: targets for early nutritional effects on epigenetic gene regulation. Mol Cell Biol 2003;23:5293-300.

13 Shi W, Bellusci S, Warburton D. Lung development and adult lung diseases. Chest 2007:132:651-6

14 Franks TJ, Colby TV, Travis WD, et al. Resident cellular components of the human lung: current knowledge and goals for research on cell phenotyping and function. Proc Am Thorac Soc 2008:5:763-6.

15 Sutherland D, Samakovlis C, Krasnow MA. branchless encodes a Drosophila FGF homolog that controls tracheal cell migration and the pattern of branching. Cell 1996;87:1091-101.

16 Bellusci S, Grindley J, Emoto H, et al. Fibroblast growth factor 10 (FGF10) and branching morphogenesis in the embryonic mouse lung. Development 1997:124:4867-78.

17 Ventura A, Young AG, Winslow MM, et al. Targeted deletion reveals essential and overlapping functions of the miR-17 through 92 family of miRNA clusters. Cell 2008;132:875-86.

18 Taganov KD, Boldin MP, Chang KJ, et al. NF-kappaB-dependent induction of microRNA miR-146, an inhibitor targeted to signaling proteins of innate immune responses. Proc Natl Acad Sci U S A 2006:103:12481-6.

19 Pottelberge GR, Mestdagh P, Bracke KR, et al. MicroRNA expression in induced sputum of smokers and patients with chronic obstructive pulmonary disease. Am J Respir Crit Care Med 2011;183:898-906.

20 Iorio MV, Piovan C, Croce CM. Interplay between microRNAs and the epigenetic machinery: an intricate network. Biochim Biophys Acta 2010;1799:694-701.

21 Harris-Johnson KS, Domyan ET, Vezina CM, et al. beta-Catenin promotes respiratory progenitor identity in mouse foregut. Proc Natl Acad Sci U S A 2009;106:16287-92

22 Konigshoff $\mathrm{M}$, Kramer M, Balsara N, et al. WNT1-inducible signaling protein-1 mediates pulmonary fibrosis in mice and is upregulated in humans with idiopathic pulmonary fibrosis. J Clin Invest 2009;119:772-87.

23 Sharma S, Tantisira K, Carey V, et al. A role for Wnt signaling genes in the pathogenesis of impaired lung function in asthma. Am J Respir Crit Care Med 2010;181:328-36.
24 Pfaff EM, Becker S, Gunther A, et al. Dickkopf proteins influence lung epithelial cell proliferation in idiopathic pulmonary fibrosis. Eur Respir J 2011;37:79-87.

25 Selman M, Pardo A, Kaminski N. Idiopathic pulmonary fibrosis: aberrant recapitulation of developmental programs? PLoS Med 2008;5:e62

26 Sears MR, Greene JM, Willan AR, et al. A longitudinal, population-based, cohort study of childhood asthma followed to adulthood. N Engl J Med 2003;349:1414-22.

27 Morgan WJ, Stern DA, Sherrill DL, et al. Outcome of asthma and wheezing in the first 6 years of life: follow-up through adolescence. Am J Respir Crit Care Med 2005:172:1253-8.

28 Stern DA, Morgan WJ, Wright AL, et al. Poor airway function in early infancy and lung function by age 22 years: a non-selective longitudinal cohort study. Lancet 2007;370:758-64.

29 Fu XW, Wood K, Spindel ER. Prenatal nicotine exposure increases GABA signaling and mucin expression in airway epithelium. Am J Respir Cell Mol Biol 2011:44:222-9.

30 Svanes C, Sunyer J, Plana E, et al. Early life origins of chronic obstructive pulmonary disease. Thorax 2010;65:14-20.

31 Murk W, Risnes KR, Bracken MB. Prenatal or early-life exposure to antibiotics and risk of childhood asthma: a systematic review. Pediatrics 2011;127: 1125-38.

32 Garcia-Marcos L, Sanchez-Solis M, Perez-Fernandez V. Early exposure to acetaminophen and allergic disorders. Curr Opin Allergy Clin Immunol 2011;11:162-73

33 Wright RJ. Perinatal stress and early life programming of lung structure and function. Biol Psychol 2011;84:46-56.

34 Nurmatov U, Devereux $G$, Sheikh $A$. Nutrients and foods for the primary prevention of asthma and allergy: systematic review and meta-analysis. J Allergy Clin Immunol 2011;127:724-33. e1-30.

35 Rusconi F, Galassi C, Forastiere F, et al. Maternal complications and procedures in pregnancy and at birth and wheezing phenotypes in children. Am J Respir Crit Care Med 2007:175:16-21

36 Latzin $\mathrm{P}$, Roosli M, Huss $\mathrm{A}$, et al. Air pollution during pregnancy and lung function in newborns: a birth cohort study. Eur Respir J 2009:33:594-603.

37 Turner SW, Palmer LJ, Rye PJ, et al. Infants with flow limitation at 4 weeks: outcome at 6 and 11 years. Am J Respir Crit Care Med 2002;165:1294-8.

38 Kurukulaaratchy RJ, Waterhouse L, Matthews SM, et al. Are influences during pregnancy associated with wheezing phenotypes during the first decade of life? Acta Paediatr 2005:94:553-8.

39 Pike KC, Crozier SR, Lucas JS, et al. Patterns of fetal and infant growth are related to atopy and wheezing disorders at age 3 years. Thorax 2010;65: 1099-106.

40 Kotecha SJ, Watkins WJ, Paranjothy $\mathrm{S}$, et al. Effect of late preterm birth on longitudinal lung spirometry in school age children and adolescents. Thorax 2012:67:54-61.

41 Aurora P, Bush A, Gustafsson P, et al. Multiple-breath washout as a marker of lung disease in preschool children with cystic fibrosis. Am J Respir Crit Care Med 2005:171:249-56.

42 Aurora P, Stanojevic S, Wade A, et al. Lung clearance index at 4 years predicts subsequent lung function in children with cystic fibrosis. Am J Respir Crit Care Med 2010;183:752-8

43 Torrens C, Poston L, Hanson MA. Transmission of raised blood pressure and endothelial dysfunction to the $\mathrm{F} 2$ generation induced by maternal protein restriction in the F0, in the absence of dietary challenge in the F1 generation. Br J Nutr 2008;100:760-6.

44 Blacquiere MJ, Timens W, van den Berg A, et al. Maternal smoking during pregnancy decreases Wnt signalling in neonatal mice. Thorax 2010;65: 553-4.

45 Brass DM, Li Z, Potts EM, et al. Maternal exposure to diesel exhaust particles during gestation heritably alters innate immune responsiveness in $\mathrm{F} 1$ and $\mathrm{f} 2$ generations. American Thoracic Society International Conference. Am J Respir Crit Care Med 2011;183:A1411.

46 Herbst T, Sichelstiel A, Schar C, et al. Dysregulation of allergic airway inflammation in the absence of microbial colonization. Am J Respir Crit Care Med 2011:184:198-205.

47 Saglani S, Mathie SA, Gregory LG, et al. Pathophysiological features of asthma develop in parallel in house dust mite-exposed neonatal mice. Am J Respir Cell Mol Biol 2009;41:281-9.

48 Saglani S, Payne DN, Zhu J, et al. Early detection of airway wall remodeling and eosinophilic inflammation in preschool wheezers. Am J Respir Crit Care Med 2007; 176:858-64.

49 Shaheen SO, Newson RB, Ring SM, et al. Prenatal and infant acetaminophen exposure, antioxidant gene polymorphisms, and childhood asthma. J Allergy Clin Immunol 2010;126:1141-8 e7. 